\title{
New ideas for glaucoma
}

Eye (2015) 29, 1241; doi:10.1038/eye.2015.153; published online 2 October 2015

Glaucoma is a term covering a heterogeneous group of conditions that have in common an irreversible and usually progressive optic neuropathy resulting in distinctive patterns of visual field loss. Primary open-angle glaucoma (POAG) is by far the most common form in the United Kingdom (UK). Around 2\% of the population over the age of 40 years have POAG, and the percentage increases to $\sim 4 \%$ at the age of 80 years. ${ }^{1}$ In the context of the ageing population, the prevalence of glaucoma will rise and become an even greater health problem. It is estimated that up to $35 \%$ of the retinal ganglion cells have already been lost before visual field defects can be detected by perimetry, and subjective awareness of field loss usually occurs later still. ${ }^{2}$ Therefore, only after the eye has already suffered significant and permanent damage is a patient likely to be commenced on treatment. In developed countries, fewer than $50 \%$ of those with glaucoma are aware of their disease. ${ }^{3}$ There is currently no available screening method that reliably predicts glaucoma prior to the occurrence of permanent damage.

In the United Kingdom, the annual cost associated with blindness due to glaucoma probably exceeds $£ 100$ million. ${ }^{1}$ The real impact of the disease is almost certainly greater, as visual field loss can have a profound impact on the quality of life of the affected patient, such as loss of driving licence even when visual acuity is relatively unaffected. In addition, treatments given for glaucoma, whether surgical or medical, can further reduce the patient's quality of life. On an individual basis, the lifestyle changes necessary when suffering from this chronic disease and the functional limitations resulting from loss of vision are immense.
A Lotery

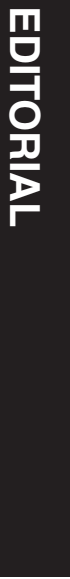

This loss of vision may be postponed or prevented by early diagnosis and appropriate treatment. However, with the current methods, early diagnosis still cannot prevent permanent visual loss. In addition, despite the best current practice, POAG often progresses, resulting in severe visual impairment to many patients. Therefore it is an important disease to study.

In this special issue of Eye, we are delighted to report the latest research related to the study of glaucoma. The papers include descriptions of novel surgical techniques, methods to improve compliance with therapy, and novel ways to assess progression of glaucoma. In addition, leading glaucoma researchers have contributed insightful reviews, which challenge the current dogma regarding the pathogenesis of glaucoma as well as highlight exciting new imaging techniques to detect glaucomatous damage at the earliest stage. Therefore, there is much in this issue to convince researchers, clinicians, and patients that glaucoma treatment can be improved. Eye is proud to contribute to this effort via publication of this work.

\section{Conflict of interest}

The author declares no conflict of interest.

\section{References}

1 Royal College of Ophthalmologists. A National Research Strategy for Ophthalmology. Royal College of Ophthalmologists: London, UK, 2002.

2 Kerrigan-Baumrind LA, Quigley HA, Pease ME, Kerrigan DF, Mitchell RS. Number of ganglion cells in glaucoma eyes compared with threshold visual field tests in the same persons. Invest Ophthalmol Vis Sci 2000; 41(3): 741-748.

3 Quigley HA. Number of people with glaucoma worldwide. Br J Ophthalmol 1996; 80(5): 389-393. A Lotery, Faculty of
Faculty of Medicine, Division of Neurosciences, University of Southampton, Southampton General Hospital, Southampton, UK

Correspondence: Medicine, Division of Neurosciences, University of Southampton, Southampton General Hospital, South Lab and Path Block, Mail Point 806, Level D, Southampton, Hampshire S016 6YD, UK Tel: +44 (0)238 079 4590/ 5049;

Fax: +44 (0)238 079 4264/ 4542.

E-mail: a.j.lotery@soton.ac.uk 